\title{
Esplenectomía laparoscópica en enfermedades hematológicas
}

\author{
Fernando Maluenda G, Patricio Burdiles P, \\ Italo Braghetto M, Attila Csendes J. \\ Laparoscopic splenectomy \\ in hematological diseases
}

Background: Idiopathic thrombocytopenic purpura (ITP) is the most common indication for elective splenectomy. The laparoscopic approach has been used over the past ten years. Aim: To report our experience with laparoscopic splenectomy. Patients and methods: Retrospective review of 27 patients subjected to splenectomy due to hematological diseases. Among them, 17 patients (78\% female, age range 17-70 years old) were subjected to a laparoscopic splenectomy. Eligibility criteria were the presence of benign disease, an informed consent by the patient, a spleen size of less than $20 \mathrm{~cm}$ by ultrasound and absence of previous surgery in the upper left quadrant. The rest of the patients were subjected to an open splenectomy. Results: Seventy one percent of patients subjected to laparoscopic splenectomy had an ITP. Mean operating time was 184 minutes. The mean spleen size was $11 \mathrm{~cm}$ and the mean weight was $186 \mathrm{~g}$ (70-450). No patient died or had complications. No patient required a conversion to an open surgery. Transfusions were not required. The median hospital stay was 3 days. Conclusions: Elective laparoscopic splenectomy is a safe and low risk surgical procedure (Rev Méd Chile 2004; 132: 189-94).

(Key Words: Laparoscopy; Purpura, thrombocytopenic; Splenectomy; Thrombocytopenia)

Recibido el 9 de junio, 2003. Aceptado en versión corregida el 13 de octubre, 2003.

Departamento de Cirugía, Hospital Clínico Universidad de Chile. Santiago

L

a esplenectomía como procedimiento terapéutico para enfermedades hematológicas, se estableció a comienzos del siglo pasado ${ }^{1}$. Por largo tiempo se efectuó a través de laparotomía, hasta que la técnica laparoscópica, ya establecida como la vía de elección para el tratamiento de colecistolitiasis, de reflujo gastroesofágico y otras patolo-

Correspondencia a: Dr. Fernando Maluenda G. Santos Dumont 999, Independencia, Santiago. Fax: 7327245. E-mail: fmaluend@ns.hospital.uchile.cl gías intraabdominales, se aplicó a la esplenectomía por primera vez en $1991^{1}$. Desde entonces se han comunicado numerosas experiencias describiendo el uso de la esplenectomía laparoscópica, en enfermedades hematológicas benignas que cursan fundamentalmente con bazo de tamaño normal o ligeramente aumentado. En Chile se reportó un caso clínico operado con éxito por esta vía en 1997 y se describió la técnica ${ }^{2}$.

En mayo de 1999, nuestro grupo comenzó a efectuar la esplenectomía por vía laparoscópica, como procedimiento de elección en el tratamiento electivo de pacientes portadores de enfermedades 
hematológicas benignas con indicación de esplenectomía.

El objetivo del presente trabajo es mostrar la experiencia inicial con la esplenectomía laparoscópica, describir sus indicaciones, contraindicaciones y la técnica quirúrgica utilizada en nuestro centro.

\section{PACIENTES y MÉTOdo}

Se trata de un trabajo retrospectivo, en base a un protocolo de intervención con criterios predefinidos.

a) Pacientes estudiados. Entre mayo de 1999 y mayo de 2003, en el Departamento de Cirugía del Hospital Clínico de la Universidad de Chile, hemos efectuado 27 esplenectomías en forma electiva. Se eligió a 17 pacientes para ser operados por vía laparoscópica y los 10 pacientes restantes fueron intervenidos mediante cirugía tradicional.

Los criterios de elección para la intervención por vía laparoscópica se enumeran a continuación:

- Criterios de inclusión: 1) Consentimiento informado por parte del paciente, previa explicación del procedimiento, sus ventajas y potenciales complicaciones; 2) Ausencia de enfermedades cardiovasculares, riesgo quirúrgico bajo (ASA I-II); 3) Tamaño esplénico preoperatorio menor a $20 \mathrm{~cm}$ de eje longitudinal, estimado por ecotomografía; 4) Diagnóstico de enfermedad hematológica benigna.

- Criterios de exclusión: 1) Rechazo por parte del paciente de la vía laparoscópica; 2) Presencia de enfermedades cardiovasculares (ASA $>$ II); 3) Tamaño esplénico $>20 \mathrm{~cm}$ de eje longitudinal; 4) Cicatriz de laparotomía previa de hemiabdomen superior; 5) Sospecha de enfermedad esplénica maligna; 6) Trauma esplénico.

No se consideró el recuento plaquetario preoperatorio ni el valor de hemoglobina en sangre entre los requisitos a cumplir .

b) Preparación preoperatoria. A todos los pacientes se les efectuó una ecotomografía abdominal, con el objetivo fundamental de evaluar tamaño esplénico, medido en su eje longitudinal y trans- verso, además de determinar la eventual presencia de colecistolitiasis asintomática. Todos los pacientes fueron inmunizados con vacuna polivalente antineumocócica (Vacuna Pneumo 23, Rhodia Merieux), al menos dos semanas previas a la intervención. El día de la intervención, todos los pacientes recibieron una dosis de corticosteroides (hidrocortisona $100 \mathrm{mg}$ ) y antibioterapia profiláctica (cefazolina $1 \mathrm{gr}$ ) vía intravenosa, una hora previa a la operación. Recuento plaquetario en el preoperatorio inmediato.

c) Técnica quirúrgica por vía laparoscópica. Todos los pacientes fueron intervenidos bajo anestesia general, en decúbito dorsal, con lateralización derecha de $30^{\circ}$ e inclinación de Fowler $25-30^{\circ}$ y piernas en abducción. En esta posición las vísceras tienden a gravitar hacia la derecha y hacia caudal, exponiendo de esta forma el bazo. Siempre se prepara mesa de instrumental estándar pues en caso de sangrado no controlable, debe procederse con celeridad a la conversión y hemostasia. La técnica laparoscópica utiliza cuatro trocares de entre 5 y $12 \mathrm{~mm}$ de diámetro, todos insertados en el área del cuadrante superior izquierdo del abdomen. La primera incisión se efectúa en región subcostal izquierda, a nivel de la línea axilar anterior, donde se inserta un trocar de $10 \mathrm{~mm}$ que permite instalar la óptica de $0^{\circ}$, siendo los otros tres trocares canales para instrumentos de trabajo. Una vez logrado el neumoperitoneo, y con una presión de trabajo de $15 \mathrm{mmHg}$, se procede a explorar la cavidad abdominal en la búsqueda dirigida de bazos supernumerarios, fundamentalmente en los sitios de mayor frecuencia descritos: hilio esplénico, ligamento gastroesplénico, omento menor y mayor, ligamento esplenocólico y mesenterio.

La esplenectomía propiamente tal, se inicia con la movilización del bazo desde su polo caudal, siguiendo con la liberación del peritoneo de su cara convexa y por la disección del ligamento gastroesplénico con ligadura y sección de los vasos cortos. A continuación, se libera su polo superior, accediendo de esta forma al hilio esplénico que habitualmente tiene una arteria y dos venas. Toda la disección y hemostasia se efectúa con electrobisturí, disector ultrasónico (Ultrashears, Autosuture-Tyco, Connecticut, USA) y hemoclips de titanio de $5 \mathrm{~mm}$. El tratamiento de 
la arteria y venas esplénicas se logra también mediante el uso de grapadoras vasculares de 2,5 mm (EndoGIA universal, Autosuture-Tyco, Connecticut, USA) con cierre de $1 \mathrm{~mm}$ para uso laparoscópico. En las últimas dos intervenciones laparoscópicas hemos introducido el uso del Sellador Vascular (Ligasure, Valleylab-Tyco, USA), que permite una disección por electrocoagulación bastante simple, rápida y segura, lo que ha contribuido a acortar el tiempo operatorio en forma ostensible.

Mediante el uso de rigurosa hemostasia el sangrado intraoperatorio es mínimo y una vez liberado el bazo de su pedículo vascular, se introduce inmediatamente en bolsa, para evitar el escurrimiento y con ello el potencial autotrasplante esplénico o esplenosis ${ }^{3}$.

La extracción de la pieza operatoria se hace a través de la ampliación de una de las incisiones por la cual se introducen los trocares, lo que permite un tamaño adecuado (35 $\mathrm{mm}$ de diámetro) para extraer el bazo in toto, especialmente en pacientes con bazo pequeño. En caso de requerir una incisión ampliada a más de $35 \mathrm{~mm}$, se tritura el bazo en forma cuidadosa dentro de la bolsa, logrando la extracción completa sin escurrimiento. En una paciente, cuyo bazo midió $18 \mathrm{~cm}$ de eje longitudinal, no fue posible introducirlo en bolsa para la extracción, debiendo ser extraído a través de una incisión de Pfannensthiel.

Se efectúa aseo, revisión de la hemostasia y nueva revisión en búsqueda de bazo supernumerario antes de concluir la intervención.

Se ha instalado un drenaje intraabdominal, de tipo aspirativo cerrado no ventilado, en cuatro pacientes debido al excesivo acúmulo de exudado seroso al terminar el acto quirúrgico.

Todos los espécimenes obtenidos han sido sometidos a estudio histopatológico de rutina en el Servicio de Anatomía Patológica de nuestro hospital para confirmar la naturaleza benigna de la enfermedad primaria.

\section{RESUltados}

Veintiuno de los 27 pacientes (78\% de la serie) eran de género femenino.
Las indicaciones de esplenectomía se listan en la Tabla 1, trombopenia, definida como recuento plaquetario $<150.000 / \mathrm{ml}$, estuvo presente en los 13 pacientes portadores de púrpura trombocitopénico y púrpura asociado a lupus, con un promedio de $70.000 / \mathrm{ml}$ plaquetas en el día de la intervención (rango 35.000 a 150.000/ml). Los otros 3 pacientes cursaron con recuento plaquetario preoperatorio normal.

Tabla 1. Indicaciones de esplenectomía laparoscópica

\begin{tabular}{|lc|}
\hline Diagnóstico & $\begin{array}{c}\text { Esplenectomía } \\
\text { laparoscópica } \\
\mathrm{n}: 17\end{array}$ \\
\hline Púrpura trombocitopénico idiopático & 12 \\
Anemia hemolítica autoinmune & 2 \\
Púrpura asociado a lupus & 1 \\
Linfoma & 2 \\
\hline
\end{tabular}

En los pacientes portadores de un púrpura, la duración promedio de la trombopenia antes de la cirugía fue de 60 meses (rango 6 a 86). Cuatro pacientes fueron calificados como esteroidorresistentes, definido como recuento plaquetario persistentemente menor de $80.000 \mathrm{plaq} / \mathrm{ml}$, después de tratamiento esteroidal con metilprednisolona de $1 \mathrm{mg} / \mathrm{kg} /$ día y ocho pacientes tuvieron indicación de esplenectomía por esteroido-dependencia, definida como recaída de la trombopenia con recuento menor a $80.000 \mathrm{plaq} / \mathrm{ml}$ después de un período de buena respuesta a los corticoides.

En la Tabla 2 se resumen las características clínicas de todos los pacientes sometidos a esplenectomía laparoscópica.

El tiempo operatorio promedio fue de $184 \mathrm{~min}$ (rango 90-300), con una clara tendencia a disminuir el tiempo quirúrgico a medida que crecía la serie, siendo los últimos pacientes intervenidos entre 90 y $120 \mathrm{~min}$.

El sangrado intraoperatorio promedio fue de $50 \mathrm{ml}$ (rango 5 a $250 \mathrm{ml}$ ). Ningún paciente 
Tabla 2. Características clínicas de todos los pacientes sometidos a esplenectomía laparoscópica

\begin{tabular}{|lc|}
\hline Características & $\begin{array}{c}\text { Esplenectomía } \\
\text { laparoscópica } \\
\text { n:16 }\end{array}$ \\
\hline Edad & $37(17-70)$ \\
Relación M:F & $1: 4$ \\
Tiempo operatorio & $184 \mathrm{~min}$ \\
Peso bazo (rango) & $186 \mathrm{gr}(70-450)$ \\
Tiempo hospitalización (rango) & 3 días (2-6) \\
Complicaciones & No \\
Transfusión G. rojos & No \\
Transfusión plaquetas & 6 \\
Bazo supernumerario & 1 \\
\hline
\end{tabular}

intervenido por vía laparoscópica requirió transfusión de glóbulos rojos. En 2 pacientes fue necesario transfundir concentrados plaquetarios en el preoperatorio inmediato (recuento plaquetario de 16.000 y 41.000) y en 4 pacientes se transfundió concentrados plaquetarios una vez ligado el pedículo esplénico.

No hubo la necesidad de convertir a cirugía tradicional a ningún paciente.

El tamaño esplénico, estimado por ecotomografía abdominal, tomando como referencia el eje longitudinal, fue de $11 \mathrm{~cm}$ en promedio (rango $9 \mathrm{a}$ $18 \mathrm{~cm}$ ). Las dimensiones del bazo extirpado por laparotomía fueron significativamente mayores.

El peso medido en pabellón, inmediatamente post extracción, varió entre 70 y 450 gramos en pacientes operados por vía laparoscópica. No se encontró una relación directa entre la longitud esplénica, medida por ecotomografía en el preoperatorio y el peso del bazo. En dos pacientes mujeres fue necesario realizar además una colecistectomía laparoscópica por presentar colelitiasis asociada.

La realimentación se reinició al día siguiente de la intervención, con buena tolerancia a la dieta líquida. No hubo vómitos ni distensión abdominal en el período postoperatorio.

El promedio de estadía intrahospitalaria fue de 3 días (rango 2 a 6). La morbilidad postoperatoria presentada fue básicamente equimosis alrededor de algunas incisiones, especialmente alrededor de la incisión umbilical en dos casos, que no requirieron ningún tipo de tratamiento por su tamaño y por ser asintomáticas. La serie no registra mortalidad.

Requerimientos analgésicos. Se usó opiáceos fraccionados vía intravenosa como analgesia postoperatoria para las primeras $24 \mathrm{~h}$, continuando con paracetamol vía oral hasta el tercer día postoperatorio.

Tabla 3. Resultados de la esplenectomía laparoscópica. Según la literatura

\begin{tabular}{|lcccccc|}
\hline Autor & año & № casos & $\begin{array}{c}\text { Complicaciones } \\
(\%)\end{array}$ & $\begin{array}{c}\text { Peso bazo } \\
(\mathrm{g})\end{array}$ & $\begin{array}{c}\text { Conversión } \\
(\%)\end{array}$ & $\begin{array}{c}\text { Tiempo Op. } \\
(\mathrm{min})\end{array}$ \\
\hline Tanoue $^{8}$ & 1999 & 35 & 11,4 & 120 & - & 204 \\
Marassi $^{4}$ & 1999 & 15 & - & 181 & - & 146 \\
Glasgow $^{10}$ & 1999 & 15 & 5 & 278 & 10 & 186 \\
Targarona $^{11}$ & 2000 & 113 & 18 & 493 & 7,4 & 153 \\
Katkhouda $^{12}$ & 2000 & 103 & 5,8 & 263 & 3,9 & 161 \\
Katkhouda1 $^{12}$ & $2000^{\mathrm{a}}$ & 461 & 8,0 & - & 8,0 & $123-210$ \\
Franciosi $^{7}$ & 2002 & 20 & 10 & - & 5,0 & 165 \\
Pérez $^{13}$ & 2002 & 11 & 11 & - & - & 102 \\
Maluenda, & 2003 & 17 & - & 186 & - & 184 \\
\hline
\end{tabular}

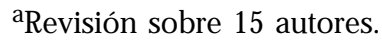


No se empleó antiinflamatorios no esteroidales en ningún paciente, así como tampoco se usó catéter peridural para analgesia en los pacientes operados por vía laparoscópica. Los pacientes intervenidos por vía clásica recibieron analgesia por vía intravenosa (opiáceos) con bomba de infusión controlada por el paciente (PCA) hasta las $72 \mathrm{~h}$ postoperatorias.

Respuesta hematológica. En pacientes portadores de púrpura trombocitopénico idiopático (12 pacientes), el recuento promedio postoperatorio fue de $113.500 \mathrm{plaq} / \mathrm{ml}$ (40.000-250.000), evaluado el mismo día de la intervención. En dos pacientes no se obtuvo respuesta inmediata, la que se verificó al día siguiente a la intervención.

Se redujo gradualmente la administración de corticosteroides hasta obtener un recuento mayor a $100.000 \mathrm{plaq} / \mathrm{ml}$, lo que se logró en promedio a los 15 días post esplenectomía.

\section{DisCUSIÓN}

Para la aceptación de una nueva técnica quirúrgica y evaluar su aplicabilidad en clínica, se debe demostrar que tiene índices de morbilidad y mortalidad en todo comparables a la técnica estándar, con igual o mejor efectividad. Existen varios estudios comparativos entre esplenectomía abierta y laparoscópica, aunque todos ellos comparan series históricas con un grupo prospectivo laparoscópico ${ }^{4-7}$. A esta última técnica se le atribuyen beneficios tales como menor dolor postoperatorio, menos complicaciones derivadas de la incisión, la rápida deambulación postoperatoria, rápida restitución al trabajo y una estadía intrahospitalaria más corta ${ }^{5-7}$.

A pesar que el beneficio de la esplenectomía laparoscópica está aún en vías de ser demostrado, nosotros hemos iniciado esta serie prospectiva de 17 pacientes, aplicando criterios de inclusión, arbitrario en un comienzo, y acotada a pacientes con bazo de tamaño normal o discretamente aumentado y a portadores de patología benigna. Hasta ahora, no hemos incluido pacientes con bazo grande, definido como bazo mayor a $20 \mathrm{~cm}$ en el eje longitudinal por ecotomografía, que para algunos grupos, constituiría una contraindicación formal para el método laparoscópico ${ }^{6}$, pero sí hemos comenzado a aplicar esta técnica a pacientes con sospecha de cuadros hematológicos malignos.

La indicación más frecuente de esplenectomía fue el púrpura trombocitopénico idiopático (PTI). Creemos que la cirugía mínimamente invasiva constituye la alternativa ideal para efectuar la esplenectomía en esta enfermedad, dado que en general cursa con bazo de tamaño normal o discretamente aumentado, con esta técnica se tiene un mínimo riesgo de sangrado y permite la extracción del bazo en bolsa, triturado, lo que no altera el estudio histopatológico, evitando así la ampliación de incisiones para la extracción de la pieza operatoria.

Hubo elevación del recuento plaquetario en todos los casos portadores de PTI al momento del alta.

En esta serie intervenida por vía laparoscópica encontramos la presencia de bazo supernumerario en un paciente, lo que puede presentarse entre 10 y $14 \%$ de los $\operatorname{casos}^{5,6}$. Concordamos en que la vía laparoscópica es una excelente técnica para esta detección, por el gran aumento óptico del campo quirúrgico que produce y por ser un procedimiento, en general, limpio, con mínimo escurrimiento, que da facilidades para observar tejido esplénico ectópico, a diferencia de la técnica abierta que, en general, es más traumática, y tiende a infiltrar con tinte hemático los tejidos vecinos, dificultando así la visión de este tipo de tejido.

A pesar que la cirrosis hepática con hipertensión portal es una de las contraindicaciones formales descritas para el método laparoscópico ${ }^{6}$, uno de nuestros pacientes tenía cirrosis hepática histológicamente demostrada por biopsia hepática, pero sin hipertensión portal, por lo que no representó un sangrado ni dificultad mayor, y no fue necesaria la conversión a cirugía clásica.

Factores de riesgo de conversión descritos, son el antecedente de laparotomías previas y fundamentalmente el tamaño esplénico grande ${ }^{6}$. Por ahora, hemos considerado excluidos de esta serie inicial a estos pacientes, hasta aumentar la casuística y con ello la experiencia, de tal forma de disminuir el riesgo de conversión a cirugía abierta.

Es importante remarcar que este procedimiento requiere de un equipo integrado de hematólogos, cirujanos y banco de sangre. Los pacientes deben ser inmunizados previamente para infec- 
ciones contra gérmenes encapsulados. El equipo quirúrgico debe tener experiencia en procedimientos laparoscópicos, dado el meticuloso cuidado que exige el manejo del pedículo esplénico y que ha sido la clave en esta serie para evitar la conversión a laparotomía de urgencia por hemorragia de este pedículo.

Aunque la serie inicial es pequeña, creemos que la esplenectomía por vía laparoscópica es una

\section{REFERENCIAS}

1. Delaitre B, Maignen B. Splenectomie par voie Laparoscopique 1. Observation. Presse Med 1991; 20: 2263.

2. Vauejos C, Torres F, González G. Esplenectomía laparoscópica. Rev Chil Cir 1997; 49: 705-7.

3. Espert J, Targarona E, Bombuy E, Setoain J, Visa J, TRIAS M ET AL. Evaluation of risk of splenosis during laparoscopic splenectomy in rat model. World J Surg 2001; 25: 882-5.

4. Marassi A, Vignali W, Zuliani W, Biguzzi E, Bergamo C, Gianotti L et al. Splenectomy for idiopathic thrombocytopenic purpura. Comparison of laparoscopic and conventional surgery. Surg Endosc 1999; 13: 17-20.

5. Targarona E, Espert J, Cerdan G, Balague C, Piulachs J, Sugrañes G et al. Effect of spleen size on splenectomy outcome. A comparison of open and laparoscopic surgery. Surg Endosc 1999; 13: $559-62$.

6. Klinger P, Tsiotos G, Giaser K, Hinder R. Laparoscopic splenectomy: evolution and current status. Surg Laparosc Endosc Percutan Tech 1999; 9: 1-8. excelente altemativa de tratamiento para los pacientes portadores de enfermedades hematológicas, especialmente el púrpura trombocitopénico idiopático. Es segura, produce muy poco dolor postoperatorio, lo que conlleva un muy bajo consumo de analgésicos, tiene una casi nula morbilidad, estadía intrahospitalaria breve y con un excelente resultado cosmético, considerando toda vez que es una patología mayoritariamente del género femenino.

7. Franciosi C, Caprotti R, Romano F, Porta G, Real G, Cоцомво G ET AL. Laparoscopic versus open splenectomy: a comparative study. Surg Laparosc Endosc Percutan Tech 2000; 10: 291-5.

8. Decker G, Milat B, GuiLon F, Atger J, Linon M. Laparoscopic splenectomy for benign and malignant hematologic diseases: 35 consecutive cases. World J Surg 1998; 22: 62-8.

9. Tanoue $\mathrm{K}$, Hashizume M, Morita M, Migoh S, Tsugawa K, Yagi S et al. Results of Laparoscopic Splenectomy for Inmune Thrombocytopenic Purpura. Am J Surg 1999; 3: 222-6.

10. Glasgow R, MulvihiL S. Laparoscopic splenectomy. World J Surg 1999; 23: 384-8.

11. Targarona E, Espert J, Bombuy E, Vidal O, Cerdan G, Artigas V et al. Complications of laparoscopic splenectomy. Arch Surg 2000; 135: 1137-40.

12. KaTKHOUda N, Mavor E. Laparoscopic splenectomy. Surg Clin of NA 2000; 80: 1285-97.

13. Pérez G, escalona A, López F, Pérez M, Crovari F, Boza C ET AL. Esplenectomía laparoscópica en pacientes con púrpura trombocitopénico idiopático. Rev Chil Cir 2002; 54: 639-43. 